\title{
Mitochondrial Crista
}

National Cancer Institute

\section{Source}

National Cancer Institute. Mitochondrial Crista. NCI Thesaurus. Code C33132.

A thin space between the outer and inner mitochondrial membranes formed by an invag ination of the inner mitochondrial membrane. 